\title{
Análisis de los proyectos del sector agropecuario financiados por Colciencias durante el año 2010
}

\section{Analysis of agricultural projects funded by Colciencias during $\mathbf{2 0 1 0}$}

\author{
Edison Suárez O, ${ }^{1}$ M.Sc, Mónica Baquero P, ${ }^{1 *}$ M.Sc. \\ ${ }^{1}$ Departamento Administrativo de Ciencia, Tecnología e Innovación - Colciencias. Programa Nacional \\ de Ciencia, Tecnología e Innovación Agropecuaria. Carrera 7 B Bis \# 132 - 28. Bogotá, Colombia. \\ *Correspondencia: mmbaquero@colciencias.gov.co
}

Recibido: Abril de 2012; Aceptado: Enero de 2013.

\section{RESUMEN}

Objetivo. Analizar los proyectos del sector agropecuario financiados por Colciencias durante el año 2010. Materiales y métodos. Se utilizó la base de datos de registro de proyectos correspondientes al Programa Nacional de Ciencia, Tecnología e Innovación Agropecuarias para identificar las propuestas presentadas, elegibles y financiadas de las convocatorias del año 2010. Los proyectos se clasificaron con base en la nomenclatura Internacional de la UNESCO para los campos de la ciencia y la tecnología. Se identificó la convocatoria, campos, disciplinas, rama productiva, entidades participantes, departamento de ejecución y montos financiados. Resultados. Durante el año 2010 Colciencias recibió un total de 4.725 propuestas para financiación, de éstas, 790 correspondieron al Programa Nacional de Ciencia, Tecnología e Innovación Agropecuaria. Las disciplinas de los proyectos financiados, fueron en su mayoría pertenecientes al subsector agrícola, seguido por el pecuario y el agroindustrial. Las universidades públicas fueron el tipo de entidad ejecutora al que se le financió un mayor número de proyectos. Los departamentos en los cuales se ejecutó la mayoría de las propuestas financiadas fueron Antioquia, Cundinamarca, Boyacá y Tolima. Conclusiones. El Programa Nacional de Ciencia Tecnología e Innovación Agropecuaria recibió el 16.7\% del total de proyectos recibidos por Colciencias en sus convocatorias del año 2010. El mayor número de proyectos recibidos, elegibles y financiables fueron del subsector agrícola y estuvieron presentados y ejecutados por universidades públicas. La inversión total de Colciencias en proyectos del sector agropecuario fue de $\$ 32.923 .594 .000$ con un aporte de contrapartida de $\$ 33.225 .740 .000$.

Palabras clave: Ciencia, innovación científica, proyecto de investigación, tecnología (Fuente: CAB). 


\section{ABSTRACT}

Objective. Identify and analyze the science, technology and innovation projects of the agricultural and livestock sector, funded by Colciencias during 2010. Materials and methods. The registration database of Colciencias was used to identify eligible and funded project proposals by the National Program of Agricultural Science, Technology and Innovation among 2010 calls. The projects were classified based on the UNESCO International nomenclature for the fields of science and technology. The variables identified for each project were: number of the call, field, discipline, subdiscipline, productive chain, participating institutions and execution department. Results. In 2010 Colciencias received a total of 4,725 research proposals for funding, of these the National Program of Agricultural Science, Technology and Innovation received 790 projects. The disciplines of the projects financed were mostly from the agricultural subsector, followed by livestock and agribusiness. Public universities were the type of entity that received most of the funding for research projects. The departments that received most of the funding from Colciencias were Antioquia, Cundinamarca, Boyaca and Tolima. Conclusions. The National Program of Agricultural Science, Technology and Innovation received $16.7 \%$ of all projects received by Colciencias calls in 2010 . Most of the projects submitted, eligible and funded were from the agricultural subsector. Most of these projects were presented and implemented by public universities. Total investment of Colciencias to fund the agricultural sector was $\$ 32,923,594$ with a matching contribution of $\$ 33.225 .740,50.2 \%$ of total project value.

Key words: Research project, science, scientific innovation, technology (Source: $C A B$ ).

\section{INTRODUCCIÓN}

El Departamento Administrativo de Ciencia, Tecnología e Innovación - Colciencias fue creado a partir de la Ley 1286 de 2009. Con esta ley se buscó transformar al Instituto Colombiano para el Desarrollo de la Ciencia y la Tecnología "Francisco José de Caldas" Colciencias para fortalecer el Sistema Nacional de Ciencia, Tecnología e Innovación (SNCTI) y a Colciencias para que la ciencia, la tecnología y la innovación fueran la base del modelo productivo. La agregación de valor a los productos y servicios de la economía nacional y el desarrollo productivo de una nueva industria nacional fueron el fin último de los cambios propuestos en esta ley (1).

Los Programas Nacionales de Ciencia y Tecnología fueron creados con el Decreto 585 de 1991 (2), y en el año 2010, con la Resolución 2040, algunos Programas Nacionales cambiaron su nombre y adicionalmente se creó uno nuevo. En la actualidad existen 12 Programas Nacionales en Colciencias: Programa Nacional de Ciencia, Tecnología e Innovación en Salud, Programa Nacional de Ciencias Básicas, Programa Nacional de Investigaciones en Energía y Minería, Programa Nacional de Ciencias, Tecnologías e Innovación en Áreas Sociales y Humanas, Programa Nacional de Electrónica, Telecomunicaciones e Informática, Programa Nacional de Ciencia, Tecnología e Innovación del Mar y de los Recursos
Hidrobiológicos, Programa Nacional de Ciencia, Tecnología e Innovación en Educación, Programa Nacional de Ciencia, Tecnología e Innovación en Ambiente, Biodiversidad y Hábitat, Programa Nacional de Ciencia, Tecnología e Innovación Agropecuarias, Programa Nacional de Biotecnología, Programa Nacional de Desarrollo Tecnológico e Innovación Industrial y Programa Nacional de Ciencia, Tecnología e Innovación en Seguridad y Defensa (3).

Actualmente Colciencias es el organismo rector del SNCTI y su función está orientada hacia la promoción de una cultura basada en la generación, la apropiación y la divulgación del conocimiento y la investigación científica, la innovación y el aprendizaje permanente para articular y enriquecer la investigación, el desarrollo científico, tecnológico y la innovación con el sector privado, en especial el sector productivo (1). Las convocatorias públicas son una de las herramientas que ofrece Colciencias con la finalidad de lograr sus objetivos. Cada convocatoria corresponde a un tipo de apoyo particular que busca movilizar recursos de cofinanciación de proyectos en actividades de ciencia, tecnología e innovación.

Durante el año 2010 Colciencias abrió un total de 21 convocatorias. Seis de ellas tuvieron propuestas específicas presentadas al Programa Nacional de Ciencia, Tecnología e Innovación 
Agropecuarias. Sin embargo, este Programa Nacional no recibió propuestas provenientes de las 27 convocatorias que abrió Colciencias durante el año 2011.

Debido a que se desconocen las líneas temáticas que actualmente están siendo financiadas por Colciencias en el sector Agropecuario, el objetivo de esta investigación fue identificar y analizar los proyectos del sector agropecuario presentados a las convocatorias del año 2010 financiados por Colciencias.

\section{MATERIALES Y MÉTODOS}

Convocatorias. Con ayuda del Sistema Integral de Gestión de Documentos - SIGP, utilizado en Colciencias, se identificaron las convocatorias publicadas durante el año 2010. Adicionalmente se utilizó la base de datos de registro de proyectos con el fin de conocer las propuestas presentadas, elegibles (aprobadas con posibilidad de financiación) y financiadas por Colciencias correspondientes a las convocatorias del año 2010. Se identificaron los proyectos correspondientes al Programa Nacional de Ciencia, Tecnología e Innovación Agropecuaria.

Campo y disciplina. Se hizo una clasificación de los proyectos con base en la nomenclatura Internacional de la UNESCO para los campos de la ciencia y la tecnología. Esta clasificación consiste en codificar las tecnologías por campos, disciplinas y subdisciplinas (4). Se buscó establecer el carácter disciplinar específico de cada uno de los proyectos.

Ramas productivas. Con base en el título y los objetivos de los proyectos identificados, se determinó la rama productiva que se beneficiaría con los resultados de la propuesta.

Entidad ejecutora. Se empleó la caracterización de las entidades determinada por Colciencias para identificar el tipo de entidad ejecutora de cada uno de los proyectos identificados.

Departamento de ejecución. Se revisó la ficha de cada uno de los proyectos financiados con el fin de identificar el departamento de ejecución indicado por el investigador principal del proyecto en el momento de enviar la propuesta a evaluación.

Presupuestos. Se empleó la ficha de los proyectos para identificar el presupuesto solicitado a Colciencias como financiación, y la contrapartida total de las entidades involucradas en el proyecto. Esta información fue introducida por el investigador principal en la propuesta evaluada.

Análisis de datos. Los datos obtenidos, una vez se obtuvo la información relacionada con las variables anteriormente mencionadas, fueron analizados utilizando Ms Excel ${ }^{\circledR}$.

\section{RESULTADOS}

Convocatorias. Durante el año 2010, Colciencias abrió 21 convocatorias públicas para financiar proyectos en Ciencia, Tecnología e Innovación en los diferentes sectores de la economía del país. Al Programa Nacional de Ciencia, Tecnología e Innovación Agropecuarias se presentaron un total de 797 propuestas distribuidas en seis Convocatorias. La tabla 1 resume el número y el objeto de la Convocatorias, así como el número de propuestas recibidas por Colciencias para todos los Programas Nacionales de Ciencia, Tecnología e Innovación. La figura 1 indica el

Tabla 1. Número de propuestas recibidas por Colciencias en Convocatorias 2010.

\begin{tabular}{lc}
\hline \multicolumn{1}{c}{ Convocatoria } & $\begin{array}{c}\text { Propuestas } \\
\text { recibidas }\end{array}$ \\
\hline $502:$ Conformar un banco de programas estratégicos & \\
y proyectos de investigación, desarrollo tecnológico e & 500 \\
innovación, en la modalidad de cofinanciación. & \\
$503:$ Conformar un banco de anteproyectos de & 3324 \\
investigación científica o tecnológica. & \\
$508:$ Conformar un banco de proyectos elegibles \\
de apoyo a la estandarización y acreditación de \\
pruebas y calibraciones de laboratorios - Estrategia de \\
transformación productiva mediante la innovación y el \\
desarrollo tecnológico. \\
515: Conformar un banco de proyectos elegibles sobre \\
misiones tecnológicas. \\
517: Conformar un banco de proyectos elegibles de \\
investigación, desarrollo tecnológico e innovación, para \\
ser financiados en la modalidad de crédito Bancoldex - \\
Colciencias con incentivo a la innovación. \\
521: Constituir un banco de proyectos de investigación \\
científica o tecnológica a partir de las recomendaciones \\
de los Consejos de los Programas Nacionales de Ciencia, \\
Tecnología e Innovación; apoyar la consolidación de \\
las capacidades nacionales en Ciencia, Tecnología e \\
Innovación; y financiar proyectos de investigación que \\
contribuyan a la generación de conocimiento científico y \\
tecnológico para el desarrollo económico y social del país. \\
\hline
\end{tabular}

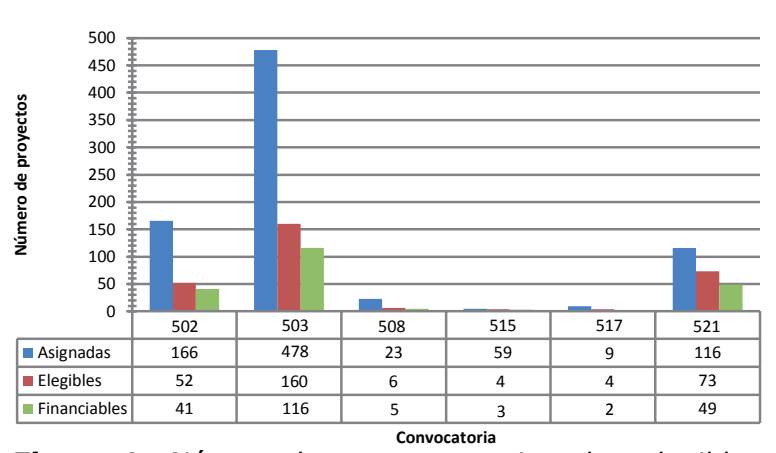

Figura 1. Número de propuestas asignadas, elegibles y financiables del Programa Nacional de Ciencia, Tecnología e Innovación Agropecuaria, 2010 por convocatoria. 
número de propuestas asignadas, elegibles y financiables del Programa Nacional de Ciencia, Tecnología e Innovación Agropecuaria.

Campo y disciplina. Las temáticas de las propuestas financiables recibidas por el Programa Nacional de Ciencia, Tecnología e Innovación Agropecuarias, fueron clasificadas de acuerdo con el subsector. La figura 2 indica el porcentaje de propuestas en ejecución (financiadas) por convocatoria clasificadas por subsector. Adicionalmente, la tabla 2 muestra el porcentaje de disciplinas por cada subsector, de acuerdo con la nomenclatura internacional de la UNESCO para los campos de ciencia y tecnología.

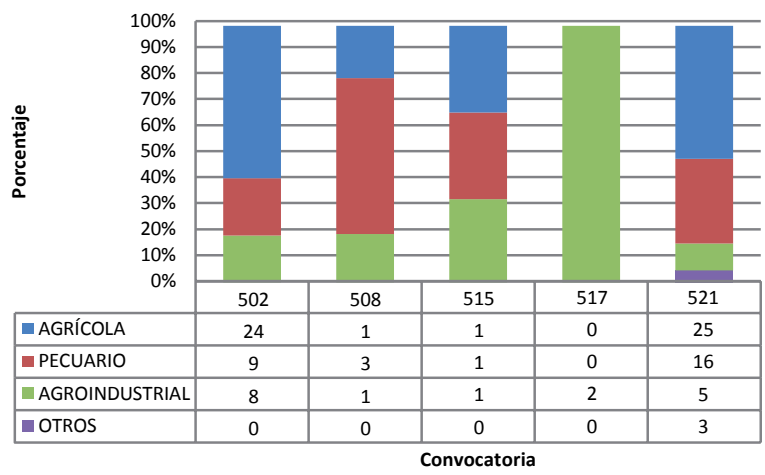

Figura 2. Porcentaje de propuestas en ejecución por convocatoria clasificadas por subsector, 2010.

Ramas productivas. La figura 3 muestra las 24 ramas productivas identificadas en los 51 proyectos del subsector agrícola que actualmente se encuentran ejecución. Estos proyectos corresponden a los financiables de las convocatorias $502,508,515,517$ y 521 .

Por otra parte, se determinó la especie de interés en los 29 proyectos identificados del subsector pecuario de las convocatorias 502, 508, 515, 517 y 521 (Figura 4). Cabe resaltar que únicamente seis proyectos de bovinos especificaron el sistema de producción del proyecto. En este sentido, se identificaron cuatro proyectos en lechería

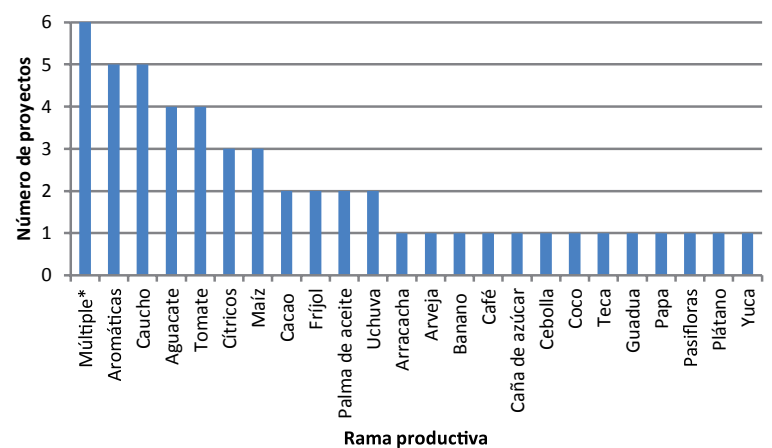

Figura 3. Número de proyectos por rama productiva identificada en los 51 proyectos del subsector agrícola.

* Proyectos cuyo objeto involucra más de una rama productiva de las identificadas (ejemplo fríjol y maíz).

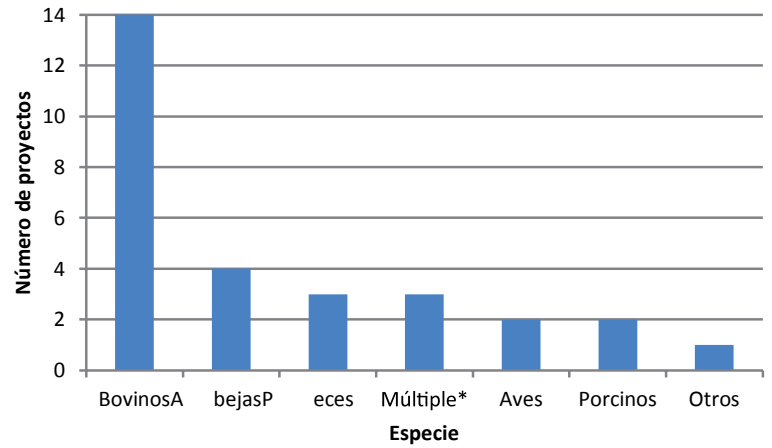

Figura 4. Especie de interés en los 29 proyectos identificados del subsector pecuario.

* Proyectos en los que hay más de una rama productiva involucrada de las identificadas (ejemplo, porcinos y bovinos).

especializada, un proyecto en ganadería de doble propósito y un proyecto en búfalos. Se agruparon como "otros" aquellos proyectos cuyas especies de interés fueron equinos, ovinos, porcinos y roedores.

En cuanto al sector agroindustrial, se identificaron siete ramas productivas (Figura 5 ) objeto de los 17 proyectos en ejecución de las convocatorias $502,508,515,517$ y 521.

Tabla 2. Porcentaje disciplinas de proyectos en ejecución por subsector por convocatoria, 2010.

\begin{tabular}{llccccc}
\hline \multicolumn{1}{c}{ Subsector } & Disciplina & $\mathbf{5 0 2}$ & $\mathbf{5 0 8}$ & $\mathbf{5 1 5}$ & $\mathbf{5 1 7}$ & $\mathbf{5 2 1}$ \\
\hline Agrícola & Agronomía & $25.00 \%$ & $0.00 \%$ & $0.00 \%$ & $0.00 \%$ & $32.00 \%$ \\
& Biología Vegetal & $4.16 \%$ & $0.00 \%$ & $0.00 \%$ & $0.00 \%$ & $16.00 \%$ \\
& Ciencia Forestal & $20.84 \%$ & $0.00 \%$ & $0.00 \%$ & $0.00 \%$ & $0.00 \%$ \\
& Entomología & $4.16 \%$ & $0.00 \%$ & $0.00 \%$ & $0.00 \%$ & $0.00 \%$ \\
& Fitopatología & $33.34 \%$ & $100.00 \%$ & $0.00 \%$ & $0.00 \%$ & $48.00 \%$ \\
& Horticultura & $12.50 \%$ & $0.00 \%$ & $100.00 \%$ & $0.00 \%$ & $4.00 \%$ \\
Pecuario & Ciencias veterinarias & $0.00 \%$ & $0.00 \%$ & $0.00 \%$ & $0.00 \%$ & $25.00 \%$ \\
& Ingeniería y tecnología del medio ambiente & $0.00 \%$ & $33.33 \%$ & $0.00 \%$ & $0.00 \%$ & $6.25 \%$ \\
& Peces y fauna silvestre & $11.11 \%$ & $0.00 \%$ & $0.00 \%$ & $0.00 \%$ & $12.50 \%$ \\
\multirow{2}{*}{ Agroindustrial } & Producción animal & $88.89 \%$ & $66.67 \%$ & $100.00 \%$ & $0.00 \%$ & $56.25 \%$ \\
& Economía sectorial & $12.50 \%$ & $0.00 \%$ & $100.00 \%$ & $0.00 \%$ & $0.00 \%$ \\
Otros & Tecnología de los alimentos & $87.50 \%$ & $100.00 \%$ & $0.00 \%$ & $100.00 \%$ & $100.00 \%$ \\
& Ciencias económicas, microbiología, ciencia política & $0.00 \%$ & $0.00 \%$ & $0.00 \%$ & $0.00 \%$ & $100.00 \%$ \\
\hline
\end{tabular}




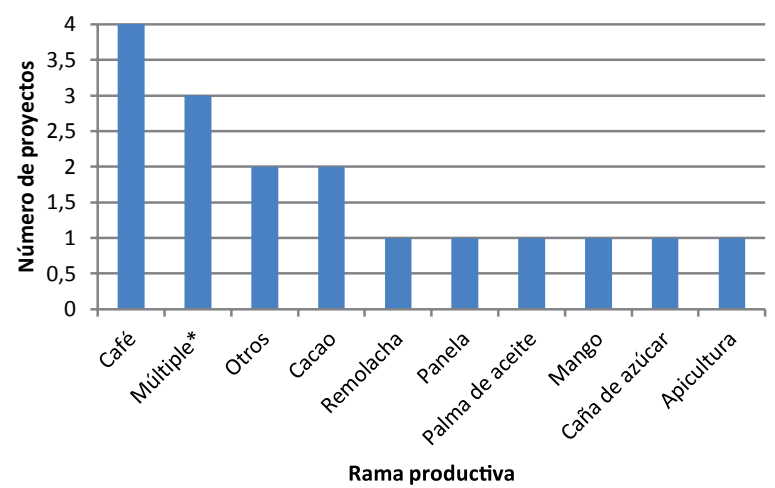

Figura 5. Ramas productivas de los proyectos en ejecución del sector agroindustrial. *Proyectos en los que hay más de una rama productiva involucrada (ejemplo, panela y caña de azúcar).

Entidad ejecutora. Adicionalmente, se hizo un análisis del tipo de entidad ejecutora tanto de los proyectos declarados elegibles, como los financiados en las convocatorias 502, 508, 515, 517 y 521.

En la figura 6 se muestran el número de proyectos elegibles de acuerdo al tipo de entidad ejecutora.

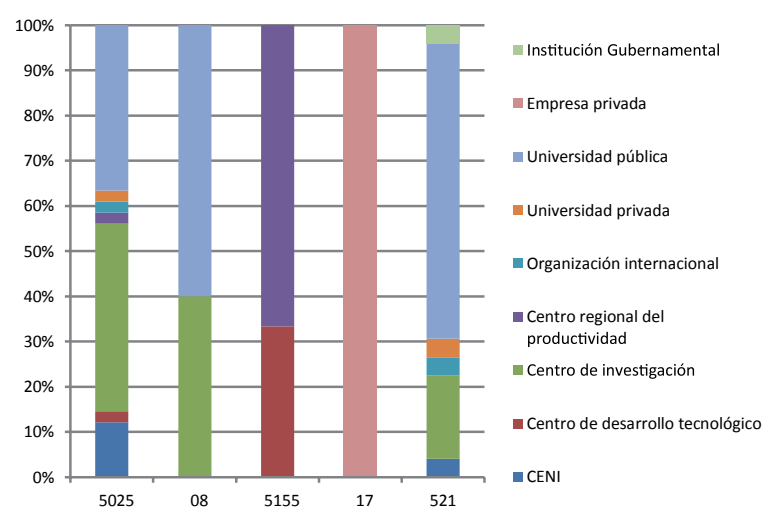

Figura 6. Porcentaje de proyectos elegibles clasificados por el tipo de entidad ejecutora.
La figura 7 indica el número de proyectos en ejecución clasificados por el tipo de entidad ejecutora.

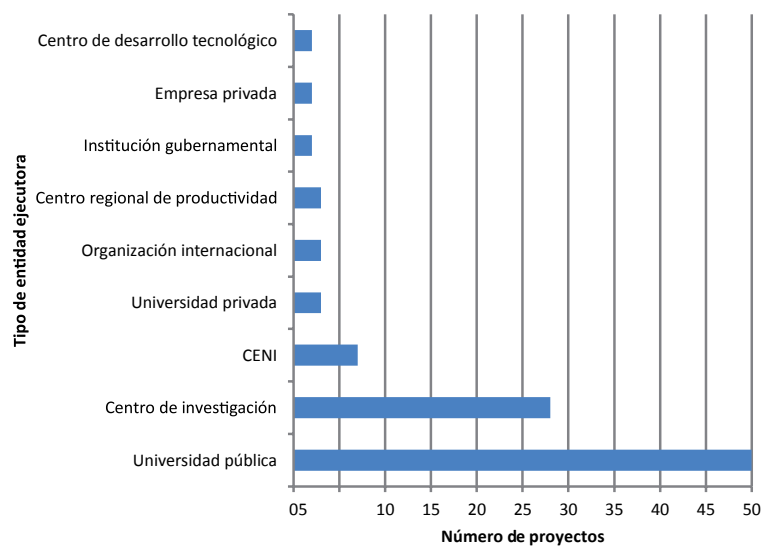

Figura 7. Número de proyectos en ejecución clasificados por el tipo de entidad ejecutora.

Departamento de ejecución. En la figura 8 se muestra el porcentaje de departamentos en los que se están ejecutando los proyectos financiados en las convocatorias 502, 508, 515, 517 y 521 . Los proyectos ejecutados en más de un departamento perteneciente a la misma región geográfica, fueron contabilizados una vez en cada uno de ellos. Adicionalmente, se consideró que un proyecto que tiene varios departamentos involucrados pertenecientes a más de dos regiones geográficas, se clasificara como de un nivel de ejecución nacional.

Presupuestos. Finalmente, Colciencias financió proyectos asignados al Programa Nacional de Ciencia, Tecnología e Innovación Agropecuaria por valor de $\$ 32.923 .594 .000$ en sus convocatorias $502,508,515,517$ y 521 .

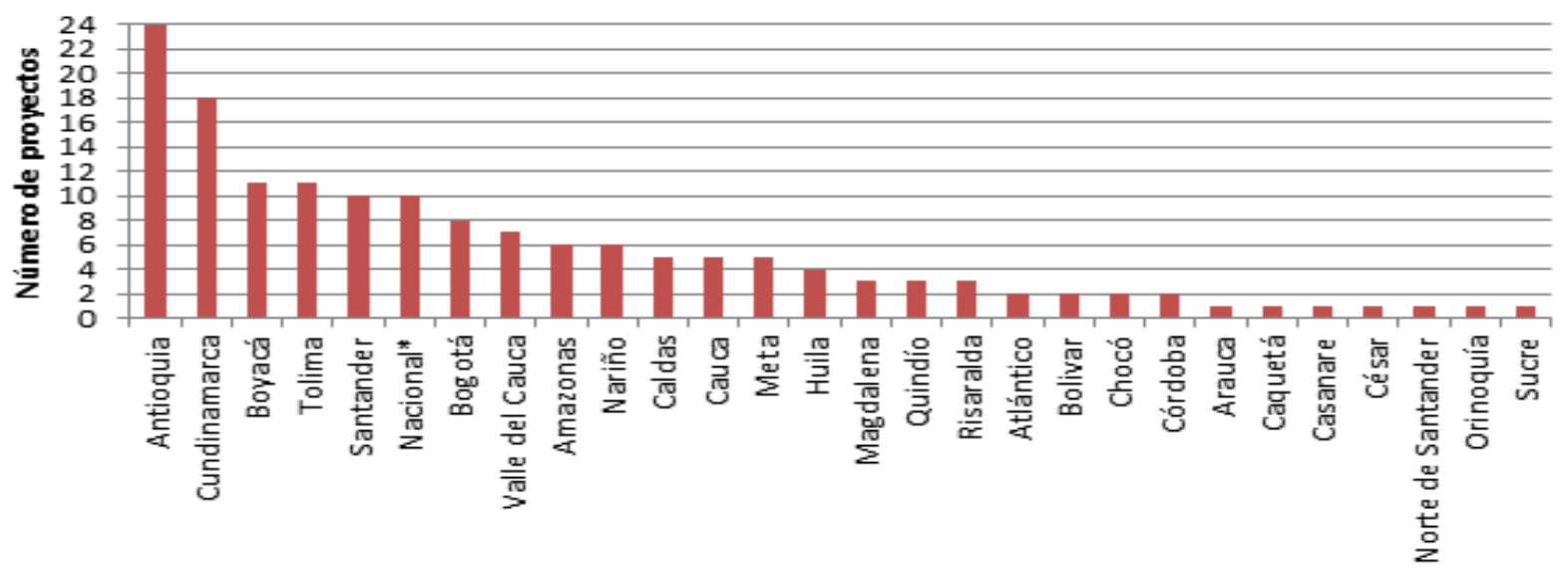

Departamento

Figura 8. Número de proyectos ejecutados por departamento.

*Proyectos ejecutados en varios departamentos pertenecientes a más de dos regiones. 
La tabla 3 especifica los montos financiados, de contrapartidas y totales por cada una de las convocatorias.

Tabla 3. Montos financiados, de contrapartidas y totales por cada una de las convocatorias en miles de pesos, 2010.

\begin{tabular}{crrrrr}
\hline Convocatoria & Financiado & $\%$ & Contrapartida & \multicolumn{1}{c}{$\%$} & \multicolumn{1}{c}{ Total } \\
\hline 502 & 21.466 .726 & 65.20 & 22.300 .698 & 67.12 & 43.767 .424 \\
508 & 422.700 & 1.28 & 383.371 & 1.15 & 806.071 \\
515 & 258.725 & 0.79 & 133.351 & 0.40 & 392.076 \\
517 & 1.919 .942 & 5.83 & 1.383 .043 & 4.16 & 3.302 .985 \\
521 & 8.855 .500 & 29.90 & 9.025 .276 & 27.16 & 17.880 .777 \\
Total & 32.923 .594 & 100 & 33.225 .740 & 100 & 66.149 .334 \\
\hline
\end{tabular}

\section{DISCUSIÓN}

EI SNCTI, cuenta con una serie de instrumentos para el fomento de la ciencia, la tecnología y la innovación, que pretenden desarrollar estrategias de apoyo formuladas en la política pública. Dichos recursos son adjudicados a través de convocatorias públicas, fundamentadas en la evaluación por pares y la revisión y recomendación de los Consejos de los Programas Nacionales de Ciencia y Tecnología (5).

Dentro de las estrategias de apoyo, el SNCTI cuenta entre otras con el apoyo a la formación avanzada de investigadores, la consolidación de capacidades para CTI, la promoción de la transformación productiva mediante la incorporación de conocimiento, la consolidación de la institucionalidad del Sistema y el fomento a la apropiación social de la ciencia, la tecnología y la innovación en la sociedad colombiana.

Cada una de estas estrategias, cuenta con un tipo o tipos particulares de apoyo, formalizados a través de convocatorias. Entre las estrategias de las convocatorias de los proyectos analizados, existen aquellas dirigidas al fortalecimiento de la productividad y la competitividad de las empresas a través del desarrollo de proyectos de investigación, desarrollo e innovación de manera articulada entre sectores productivos y académicos e investigativos (convocatoria 502). De manera individual por parte de la empresas mediante un crédito con incentivo a la innovación (convocatoria 517). Además de convocatorias orientadas a la generación de conocimiento científico o tecnológico para el desarrollo económico y social, dirigida a grupos de investigación de diferentes instituciones (Convocatorias 503 y 521).

Por otra parte, la convocatoria 508 trató de apoyar la estandarización y acreditación de pruebas en los laboratorios de las universidades y Centros de Investigación y Desarrollo Tecnológico del país. Finalmente, la convocatoria 515 trató de establecer una alianza entre empresas y centros o universidades, para la realización de misiones tecnológicas con el objetivo de identificar fuentes y oportunidades para transferencia de tecnología y modalidades de cooperación empresarial orientadas a la innovación.

El análisis de los proyectos, mostró que de las 4.725 propuestas recibidas en Colciencias, el $70.3 \%$ correspondió a los anteproyectos de investigación de la convocatoria 503, base para la formulación de los proyectos presentados a la convocatoria 521, que tal y como se muestra en los resultados, fue la convocatoria que recibió la mayor cantidad de proyectos (14.8\%). Teniendo en cuenta que la convocatoria 502 recibió 500 proyectos, se evidenció que existe una mayor dinámica en las propuestas de investigación, Desarrollo e Innovación, que en las iniciativas relacionadas con el fortalecimiento de capacidades a través de la cofinanciación de pruebas de laboratorio y de misiones tecnológicas.

Con relación a los proyectos presentados y financiados por Colciencias en el ámbito del Programa Nacional de Ciencia, Tecnología e Innovación Agropecuarias, se observó un comportamiento similar siendo la convocatoria de anteproyectos de investigación (503) la que tuvo un mayor número de propuestas recibidas, seguida por el mecanismo de cofinanciación (502) y en tercer lugar la convocatoria 521 que como ya se explicó, se basó en los anteproyectos elegibles. Es importante mencionar que en el Programa Nacional de Ciencia, Tecnología e Innovación Agropecuarias se recibieron el 33.2\% de los proyectos en la convocatoria 502 , el $15.1 \%$ de los anteproyectos de investigación (503) y el $16.5 \%$ de los proyectos en la convocatoria 521, un número muy importante, teniendo en cuenta que esta convocatoria se dirigió únicamente a 10 Programas Nacionales de Ciencia y Tecnología.

De los 12 Programas Nacionales, el Programa Nacional de Ciencia Tecnología e Innovación Agropecuaria recibió el $16.7 \%$ de los proyectos. Este resultado indica que los grupos de investigación que tienen como eje de trabajo la ciencia y la tecnología agropecuaria, presentan una dinámica importante con relación a los grupos de otros ámbitos científicos y tecnológicos. Sin embargo, según el Observatorio Colombiano de Ciencia y Tecnología (OCyT) para el año 2010 se identificó un total de 4.074 grupos de investigación activos clasificados por Colciencias; de los cuales el 5.25\% (214 grupos) 
correspondieron al Programa Nacional de Ciencia, Tecnología e Innovación Agropecuaria (6). Se recomienda realizar un trabajo minucioso basado en los grupos de investigación adscritos a los 12 Programas Nacionales cuyo fin sea correlacionar las propuestas financiadas con los ejes de trabajo de los grupos de investigación.

Con relación a los proyectos considerados elegibles, el mayor porcentaje se identificó en las propuestas presentadas a la convocatoria 515 (acreditación de laboratorios) en la que de cinco proyectos se consideraron elegibles cuatro, es decir, el $80 \%$, seguido por la convocatoria 521 (proyectos de investigación) en la que fueron elegibles el $63 \%$ de los proyectos. La convocatoria 502 (cofinanciación) tuvo un porcentaje de elegibilidad del $31.3 \%$. Es importante mencionar que el porcentaje de proyectos financiables, sólo llegó al $42.2 \%$ en la convocatoria 521 (monto financiado $\$ 8.855 .500 .343$ ) y al $25 \%$ en la convocatoria 502 (monto financiado $\$ 21.466 .726 .206$ ), la cual demuestra, que los recursos existentes para financiar proyectos en ciencia, tecnología e innovación agropecuaria, son insuficientes para atender la demanda de proyectos que cumplen con los criterios de elegibilidad en cada uno de los instrumentos de fomento analizados.

Con respecto a los subsectores beneficiados con proyectos financiados en estas convocatorias, los resultados muestran que los proyectos de índole agrícola, tuvieron un mayor porcentaje de recursos aportados, con el $58.5 \%$ de los proyectos financiados en la convocatoria 502 y el $51 \%$ en la convocatoria 521 , llama la atención el hecho de que el $100 \%$ (dos proyectos) de los financiados en la convocatoria 517 son del ámbito agroindustrial.

El subsector pecuario presentó un comportamiento promisorio con el $22 \%$ financiado en 502 $y$ el $32.6 \%$ en 521 , frente al 19.5 y $10.2 \%$ respectivamente en el ámbito agroindustrial.

En la convocatoria 502, las disciplinas según la nomenclatura Internacional de la UNESCO, con mayor porcentaje de proyectos financiados fueron en el subsector agrícola, la fitopatología con $33.3 \%$, la agronomía con $25 \%$ y las ciencias forestales con el $20.8 \%$. En el subsector pecuario, la mayor proporción de financiación la tuvieron los proyectos relacionados con la producción animal $(88.9 \%)$ y en agroindustria la tecnología de los alimentos $(87.5 \%)$.

La convocatoria 521 presentó un comportamiento similar, aunque no se financiaron proyectos en ciencias forestales y por el contrario, el $25 \%$ de los proyectos financiados en el subsector pecuario correspondieron a ciencias veterinarias. En el tema agroindustrial, en esta convocatoria el $100 \%$ de los proyectos financiados correspondió a tecnología de los alimentos.

Las ramas productivas principales identificadas en las cuales se aprobaron proyectos de las diferentes convocatorias, son el en sector agrícola las de aguacate, aromáticas, caucho, cítricos, maíz y tomate, se debe destacar 6 proyectos financiados en la categoría múltiple, la cual se refiere a proyectos en los que hay más de una cadena productiva involucrada.

En el subsector pecuario se determinó que las especies de interés con mayor cantidad de proyectos financiados fueron los bovinos con 14 proyectos, seguido de las abejas con cuatro y los peces con tres proyectos. Hay que mencionar que se financiaron tres proyectos de carácter múltiple, es decir en los cuales está más de una especie involucrada (bovinos/porcinos, bovinos/ ovinos y caprinos/ovinos).

En el ámbito agroindustrial, las ramas productivas con mayor presencia en las propuestas financiadas fueron el café y el cacao. Cabe resaltar que en esta categoría se financiaron tres proyectos catalogados como múltiples (fríjol/arveja, arroz) café/aguacate y maíz/fríjol) y dos como otros (pasifloras y caucho).

Las entidades ejecutoras principales fueron las universidades públicas con $50 \%$ (convocatorias 502,508 y 521) de los proyectos financiados por el Programa Nacional de Ciencia Tecnología e Innovación Agropecuaria, seguidas por los centros de investigación con el $28 \%$ y los CENI (Centros de investigación y desarrollo adscritos a gremios de la producción agropecuaria) con el $7 \%$.

Los departamentos en los cuales se ejecutó la mayor parte de las propuestas financiadas fueron Antioquia 16\%, Cundinamarca $12 \%$, Boyacá y Tolima $8 \%$, seguidos con una menor participación de Santander y Bogotá con $6.5 \%$ y $5.2 \%$ respectivamente. Cabe mencionar que los proyectos considerados de ejecución nacional (más de dos regiones geográficas) alcanzaron el $6.5 \%$. Lo anterior muestra una participación especial de departamentos que se han caracterizado por sus bajas capacidades científicas, tecnológicas y de innovación, dado que según el Documento Conpes 3582 de 2009, las mayores capacidades en cuanto a grupos de investigación y centros de desarrollo tecnológico, se concentran en Bogotá y Antioquia (7). 
El valor financiado por Colciencias para los proyectos presentados y declarados elegibles en el Programa Nacional de Ciencia Tecnología e Innovación Agropecuaria en las convocatorias del año 2010 fue de $\$ 32.923 .594 .000$ con un aporte de contrapartida de $\$ 33.225 .740 .000$ correspondiente al $50.2 \%$ del valor total de los proyectos. De este monto, el $65.2 \%$ fue aportado por Colciencias para la convocatoria 502, seguido del $26.8 \%$ para la convocatoria 521 .

En conclusión, durante el año 2010 el Programa Nacional de Ciencia Tecnología e Innovación Agropecuaria recibió 790 proyectos (16.7\% del total de proyectos recibidos por Colciencias en sus convocatorias del año 2010). La mayor parte de los proyectos financiados del subsector agrícola involucró más de una rama productiva, mientras que los bovinos y el café fueron las ramas productivas con un mayor número de proyectos financiados para los subsectores pecuario y agroindustrial, respectivamente. El mayor número de proyectos recibidos, elegibles y financiables fueron del subsector agrícola y estuvieron presentados y ejecutados por universidades públicas. Los departamentos que recibieron mayor financiación de los proyectos aprobados fueron: Antioquia, Cundinamarca, Boyacá y Tolima. Finalmente, el sector agropecuario se benefició con una inversión total de $\$ 66.149 .334 .000$ para proyectos de ciencia, tecnología e innovación; de este valor Colciencias financió el $49.8 \%$.

\section{REFERENCIAS}

1. Ley 1286 de 23 de enero de 2009, por la cual se modifica la Ley 29 de 1990, se transforma a Colciencias en Departamento Administrativo, se fortalece el Sistema Nacional de Ciencia, Tecnología e Innovación en Colombia y se dictan otras disposiciones. Bogotá, Colombia: Diario Oficial (47241); 2009.

2. Decreto 585 de 26 de febrero de 1991, por el cual se crea el Consejo Nacional de Ciencia y Tecnología, se reorganiza el Instituto Colombiano para el Desarrollo de la Ciencia y la Tecnología-Colcienciasy se dictan otras disposiciones. Bogotá, Colombia: Diario Oficial (39702); 1991.

3. Resolución 2040 de Colciencias, de 29 de diciembre de 2010, por la cual se establece la nueva denominación y composición de los Consejos de los Programas Nacionales de Ciencia, Tecnología e Innovación, se crea un nuevo programa y se dictan otras disposiciones. Bogotá, Colombia: Colciencias; 2010. URL Disponible en: http://www.colciencias.gov.co/sites/ default/files/upload/reglamentacion/ res_02040-2010__denominacion__y__ composicion_de_los_consejos.pdf
4. Organización de las Naciones Unidas para la Educación, la Ciencia y la Cultura. Proyecto de Nomenclatura Internacional Normalizada Relativa a la Ciencia y la Tecnología. Paris: UNESCO, 1989 URL Disponible en: http://unesdoc.unesco. org/images/0008/ 000829/082946sb.pdf

5. Instituto Colombiano para el Desarrollo de la Ciencia y la Tecnología "Francisco José de Caldas" - Colciencias. Colombia construye y siembra futuro. Política Nacional de Fomento a la Investigación y la Innovación. Bogotá, Colombia: Panamericana Formas e Impresos S.A; 2008.

6. Salazar M. Indicadores de ciencia y tecnología, Colombia 2011. Bogotá: Observatorio Nacional de Ciencia y Tecnología (OCyT); 2011.

7. Documento Conpes 3582 de 2009. Política Nacional de Ciencia, Tecnología e Innovación. Bogotá, Colombia: Departamento Nacional de Planeación; 2009. URL Disponible http://www.dnp. gov.co/Portals/0/archivos/documentos/ Subdireccion/Conpes/3582.pdf 